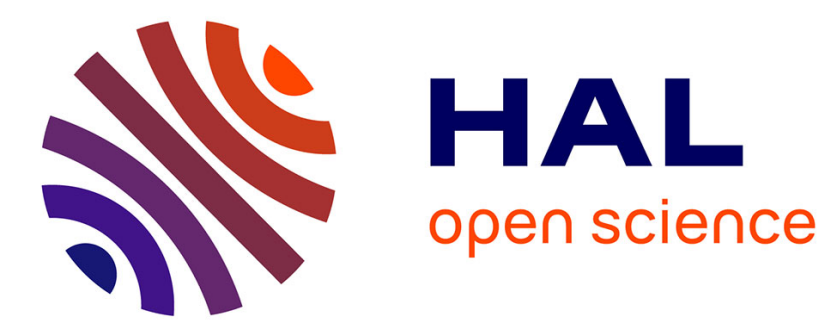

\title{
Cycles of cooperation and free-riding in social systems
}

Yiping Ma, Sebastian Gonçalves, Sylvain Mignot, Jean-Pierre Nadal, Mirta B.

Gordon

\section{To cite this version:}

Yiping Ma, Sebastian Gonçalves, Sylvain Mignot, Jean-Pierre Nadal, Mirta B. Gordon. Cycles of cooperation and free-riding in social systems. 2009. hal-00349642

\section{HAL Id: hal-00349642 \\ https://hal.science/hal-00349642}

Preprint submitted on 3 Jan 2009

HAL is a multi-disciplinary open access archive for the deposit and dissemination of scientific research documents, whether they are published or not. The documents may come from teaching and research institutions in France or abroad, or from public or private research centers.
L'archive ouverte pluridisciplinaire HAL, est destinée au dépôt et à la diffusion de documents scientifiques de niveau recherche, publiés ou non, émanant des établissements d'enseignement et de recherche français ou étrangers, des laboratoires publics ou privés. 


\title{
Cycles of cooperation and free-riding in social systems
}

\author{
Yiping $\mathrm{Ma}^{(\mathrm{a}) *}$, Sebastian Gonçalves ${ }^{(\mathrm{a})}$, Sylvain Mignot ${ }^{(\mathrm{a})}$, \\ Jean-Pierre Nadal ${ }^{(\mathrm{b}, \mathrm{c})}$ and Mirta B. Gordon ${ }^{(\mathrm{a}) \ddagger}$ \\ (a) Laboratoire TIMC-IMAG, UMR 5525 CNRS-UJF-INPG \\ Université Joseph Fourier, Grenoble \\ (b) Centre d'Analyse et Mathématique Sociales (UMR 8557 CNRS-EHESS), \\ Ecole des Hautes Etudes en Sciences Sociales, Paris \\ ${ }^{(c)}$ Laboratoire de Physique Statistique (UMR 8550 CNRS-ENS-Paris 6-Paris 7), \\ Ecole Normale Supérieure, Paris
}

January 2, 2009

\begin{abstract}
Basic evidences on non-profit making and other forms of benevolent-based organizations reveal a rough partition of members between some pure consumers of the public good (free-riders) and benevolent individuals (cooperators). We study the relationship between the community size and the level of cooperation in a simple model where the utility of joining the community is proportional to its size. We assume an idiosyncratic willingness to join the community ; cooperation bears a fixed cost while free-riding bears a (moral) idiosyncratic cost proportional to the fraction of cooperators. We show that the system presents two types of equilibria: fixed points (Nash equilibria) with a mixture of cooperators and free-riders and cycles where the size of the community, as well as the proportion of cooperators and free-riders, vary periodically.

Key-Words: Collective Systems, Complex Adaptive Systems, Social Interactions, Cycles.
\end{abstract}

\section{Introduction}

The aim of this paper is to study the relationship between the growth of a group - or community - and the quality of cooperation within this group. This concerns cooperative organizations, usually studied by anthropologists, volunteer organizations like non-profit associations and charities, informal

\footnotetext{
*Present address: Department of Physics, University of California, Berkeley, California 94720 USA

${ }^{\dagger}$ Permanent address: Instituto de Fisica, UFRGS, Porto Alegre, Brazil

${ }^{\ddagger}$ Corresponding author: mirta.gordon@imag.fr
} 
groups in the internet such as knowledge communities, open-source software communities, and many others.

Generally, contributing to the aim of the community bears some cost. But free-riding on the cooperators' efforts may also be a burden because such behavior is contrary to the social norms generally accepted in such communities and may be felt as a failure of personal morality. Thus, even if membership is voluntary, the members of such associations face a social dilemma, because they may benefit of their participation without contributing to the social good (or the common cause). Actually, a polymorphic configuration where cooperators and free riders coexist seems to be a stable form of organization. Clearly, if all the individuals shirk, everybody gets worse and the community may even disappear.

There is a vast literature on social dilemmas. Olson [1] and early work on the Prisoner Dilemma, a game theoretical formulation of this type of problems, predicted that selfish individuals have no interest to cooperate in the production of a public good, and as a result, at equilibrium all the players should be free-riders. However, many experimental economic studies (see [2] for an extensive survey) do not support this claim. Although a stable cooperation is rarely attained in finitely repeated public good games, there is substantial cooperation on the average, at least in the first periods. More generally, reciprocity, reputation-based altruism and sanctions are mechanisms that may help high and stable cooperation levels to emerge among selfish individuals. They give raise to social norms, which are standards of behaviors based on shared beliefs of how individuals ought to behave. Individuals may value approval from their peers to a generous contribution from their part. They may also value negatively their own free-riding behavior when peers contribute. All these mechanisms have proved efficient in increasing the cooperation level in experiments. Altruistic enforcement may be provided by the players after each period [3] or by external (third party) players [4]. In finitely repeated games [5], they generally contribute to increase the cooperation rates but do not succeed to achieve complete cooperation. Although these experiments do not explain why some individuals are willing to bear the cost of punishing, they show that such individuals exist.

The experimental results are usually presented as averages of the individuals' behaviors over several groups playing under similar experimental conditions. However, this average may hide quite complex individual behaviors. In a public goods experiment Hichri and Kirman [6] showed that, despite very smooth trends of average contributions, individual groups behaviors may have a very large volatility, with oscillations that become completely smeared-out after averaging. Thus, the actual behavior of individuals in public goods games may be much more complex than usually described. Another evidence is the indirect reciprocity game [7], where there is an asymmetry in the social connexions built-in by the oriented ring of in- 
teractions. Although the aggregate behavior seems almost monotonic along the periods (besides the break at which the protocol of the game -the decision dynamics- changes from parallel to sequential), the individual behavior (the amount invested at each decision conditional to the received amount) is indeed highly oscillatory. However, little attention has been paid to these oscillatory phenomena in the literature of public goods so far.

Theoretically, besides some simple games, which present cycles clearly related to their payoffs structures (like Rock, Paper and Scissors and similar games [8]) there are few examples of oscillatory behavior in large systems in the literature. Huberman and Glance [9] have shown that in a public goods problem with large populations, cycles and even chaos may be the asymptotic behavior of the system when individuals adopt particular decision strategies. In particular, if there are opportunistic individuals having a non-monotonic probability of cooperation (cooperate when there are few cooperators but free-ride if the fraction of cooperators increases above a threshold) cycles in the fraction of cooperators may exist. More recently [10] it has been shown that finite populations may oscillate between three types of strategies when playing the prisonner's dilemma. These are cycles in the strategy space, but do not necessarily give raise to cycles in the decisions or actual payoffs of the players. However, such cycles disappear in the limit of very large systems. In fact, large systems of interacting agents with the usual best response dynamics may present a cyclic behavior at equilibrium whenever the interactions are non-symmetric. Iori and Koulovassilopoulos [11] have shown that a simple model of consumers with social interactions similar to the one proposed by Durlauf [12] present oscillations in the consumption level provided that the interactions are sufficiently asymmetric. This generalizes well known results in the statistical physics literature, where it is known that the attractors of systems with completely asymmetrical interactions (i.e. the influence of individual $i$ on individual $j$ and that of $j$ on $i$ have opposite signs) may be cycles of order 2 or 4 , depending on the decisions dynamics. Moreover, even with symmetric interactions, if all the individuals make their decisions at the same time (parallel dynamics) cycles of order two cannot be excluded (although they are seldom observed in computer simulations).

In this paper we raise the question of the types of equilibria that may appear in the case of heterogeneous populations, when the heterogeneity is related to both the willingness to join the community, and the strength of the moral burden felt by free-riders. Besides the possible fixed point Nash equilibria, we also look for the possible existence of oscillatory behavior, i.e. cycles. More specifically, intuition suggests that a community is more likely to be stable if the amount of cooperation within the group is high. However, the conditions under which a polymorphic community with both free-riders and cooperators may exist in equilibrium, is still an open question. In particular, is there some threshold of cooperation level beyond which 
such a community may be stabilized? Another question is whether, from a collective point of view, it is better to have a large community with a large proportion of free-riders or a relatively small one but with a majority of cooperators.

We consider the model proposed in [13], which is an extension of the basic economic model of binary choices with externality $[12,14,15,16]$. It considers a population of interacting individuals with idiosyncratic preferences that have to decide whether to join a peers organization and cooperate, whether to join it without cooperating (free-riding) or whether not to join it at all. Cooperators bear a fixed cost, but free-riding has an idiosyncratic cost due to the social disapproval [5] experienced by a free-rider when facing cooperators.

The paper is organized as follows: section 2 summarizes the details of the model. Section 3 is devoted to the study of the different possible equilibria in the limit of an infinite population under parallel and random sequential dynamics. In section 4 the results are applied to a particularly interesting case. We show that beyond the usual Nash equilibria, that correspond to fixed points, cycles are also expected. The theoretical analysis is compared with computer simulations, showing that cycles persist in finite size systems. The results are discussed in section 6 , and we conclude in section 7 .

\section{The model of choice with social interactions}

We consider a population of $N$ agents in which each individual $i$ must choose one among the following three possibilities:

- to join the community and cooperate $\left(s_{i}=1\right)$

- to join the community and free-ride $\left(s_{i}=-1\right)$

- not to join the community $\left(s_{i}=0\right)$

In the following, we denote $\eta_{c}$ and $\eta_{f}$ the population fraction of cooperators and of free-riders respectively,

$$
\begin{gathered}
\eta_{c}=\frac{1}{N} \sum_{k=1}^{N} \delta_{s_{k}, 1} \\
\eta_{f}=\frac{1}{N} \sum_{k=1}^{N} \delta_{s_{k},-1},
\end{gathered}
$$

where $\delta_{s, s^{\prime}}$ denotes the Kronecker delta (=1 if $s=s^{\prime}, 0$ otherwise). The total fraction of individuals in the population that belong to the organization is $\eta_{c}+\eta_{f}$.

We assume that each agent $i$ has a private estimate $H_{i}$ of the value of the community, that determines his idiosyncratic willingness to join (IWJ) it. 
Besides, the community value depends on its social composition, increasing proportionally to the fraction of individuals that join it. We assume that this social component takes the simple form of a badwagon effect, given by $(J+G) \eta_{c}+J \eta_{f}$ with $J \geq 0$ and $G \geq 0$. The term $(J+G) \eta_{c}$ means that the social benefit produced by each cooperator is $(J+G) / N$, while it is only $J / N$ per free-rider. With these hypothesis, free-riders also help making the community attractive, although to a lesser extent than cooperators. Therefore, the value of the community for an individual $i$ is $H_{i}+(J+$ $G) \eta_{c}+J \eta_{f}$

Cooperators bear a fixed $\operatorname{cost} C \geq 0$ that we assume constant, the same for everyone. Free-riders in contrast support a cost proportional to the number of cooperators, weighted by an idiosyncratic factor $X_{i} \geq 0$. This cost may be interpreted as a moral burden: $X_{i}$ reflects the importance given by $i$ to the disapproval of cooperating peers.

The utility or surplus $U_{i}$ of individual $i$ depends on his own choice as well as on the choices of the others:

$$
\begin{aligned}
U_{i}\left(s_{i}=+1 \mid \eta_{c}, \eta_{f}\right) & =H_{i}+(J+G) \eta_{c}+J \eta_{f}-C \\
U_{i}\left(s_{i}=-1 \mid \eta_{c}, \eta_{f}\right) & =H_{i}+(J+G) \eta_{c}+J \eta_{f}-X_{i} \eta_{c} \\
U_{i}\left(s_{i}=0 \mid \eta_{c}, \eta_{f}\right) & =0 .
\end{aligned}
$$

We assume that individuals have a myopic behavior, i.e. at each time step every agent makes the choice which maximizes his utility, estimated from the current observed values of the fractions of cooperators and free-riders.

This model is different from the usual games considered in experimental economics of public goods. In the latter, an amount proportional to the individual contributions ( $\mathrm{C} \eta$ in our model) is equally distributed among the members. Such models are suited for explaining cooperation in small groups whose members are remunerated according to the collective output. Here we consider large organizations whose value is strongly related to their collective action, but whose production is not redistributed among the members. This is the case of non-profit organizations and other kinds of communities cited in the introduction. The value of the community is not related to the costs bore by its members, but to their number: larger communities are more attractive.

It is convenient to write the surplus of $i$ as follows:

$$
U_{i}\left(s_{i} \mid \mathbf{s}_{-i}\right)=\left(A_{i}+B_{i}\right) \delta_{s_{i}, 1}+A_{i} \delta_{s_{i},-1}
$$

where $\mathbf{s}_{-i}$ represents the choices of the other agents. $A_{i}$ is the surplus of joining the community being free-rider, and $B_{i}$ is the bonus for cooperating:

$$
\begin{aligned}
A_{i} & =H_{i}+\left(J+G-X_{i}\right) \eta_{c}+J \eta_{f}, \\
B_{i} & =X_{i} \eta_{c}-C .
\end{aligned}
$$


Then, the best response of agent $i$ given the choices of the other agents may be written as follows:

$$
\begin{aligned}
& s_{i}=1 \Longleftrightarrow A_{i}+B_{i}>0 \text { and } B_{i}>0 ; \\
& s_{i}=-1 \Longleftrightarrow A_{i}>0 \text { and } B_{i}<0 ; \\
& s_{i}=0 \text { otherwise }
\end{aligned}
$$

Notice that, by eq. (6a) we may have $A_{i}<0$ compensated by a positive $B_{i}$, making thus profitable for $i$ to join the community provided that he cooperates. This may be the case for highly moral individuals, i.e. having large weights $X_{i}$.

One can note that in this model the social interactions are not symmetric because of the idiosyncratic weights $X_{i}$. More precisely, the interaction between cooperators is symmetric: if $i$ and $j$ are both cooperators, they contribute equally to their respective utilities, by $(J+G) / N$. But whenever one free-rider at least is involved, interactions are non-symmetric. For example, if $i$ cooperates and $j$ free-rides, the contribution of $i$ to $j$ 's utility is $\left(J+G-X_{j}\right) / N$ while the contribution of $j$ to $i$ 's utility is $J / N$. Due to this dissymmetry there is no general result ensuring that the dynamics converges to fixed point solutions. These are guaranteed only for symmetric interactions (see [11] for a discussion in a case of social systems). Thus, cyclic attractors and even chaos may exist. Notice also that some agents may have $X_{i}<J+G$, and others $X_{i}>J+G$ : that is, there may be a mixture of positive and negative influences. In this case, reminiscent of spin-glass systems in physics, there may be a very large number of equilibria for some range of parameters. Although multiple equilibria do appear as the generic situation in our model, we will see that their number remains small, at least for the particular case of global interactions considered here.

In our analysis we assume that the idiosyncratic terms $H_{i}$ and $X_{i}$ are randomly distributed among the population, with averages $H$ and $X$ and variances $\sigma_{H}$ and $\sigma_{X}$ respectively. For the analysis it is convenient to work with dimensionless variables. To this end we divide all the parameters entering the utility by a quantity that plays the role of unit of measurement ${ }^{1}$, that we denote $\beta$. Thus we write

$$
H_{i}=\beta\left(h+y_{i}\right)
$$

where $H \equiv \beta h$ stands for the population's average willingness-to-join the community. Thus, $y_{i}$ is a dimensionless random variable; its probability density function (pdf) $f_{Y}(y)$ has zero mean and variance $\sigma_{H} / \beta$. We denote the parameters of the model in units of $\beta$ with small characters:

$$
h=\frac{H}{\beta}, j=\frac{J}{\beta}, g=\frac{G}{\beta}, c=\frac{C}{\beta}, x_{i}=\frac{X_{i}}{\beta},
$$

\footnotetext{
${ }^{1}$ We may choose any convenient unit, like $H, \sigma_{H}$, homogeneous to a utility.
} 
and correspondingly, $a_{i}=A_{i} / \beta, b_{i}=B_{i} / \beta$.

Hereafter we consider the equilibria corresponding to an iterative best response dynamics: starting from an arbitrary initial condition each agent chooses the strategy that maximizes his surplus. Agents may either make their decisions simultaneously (parallel dynamics) or one after the other in an arbitrary order (random sequential dynamics).

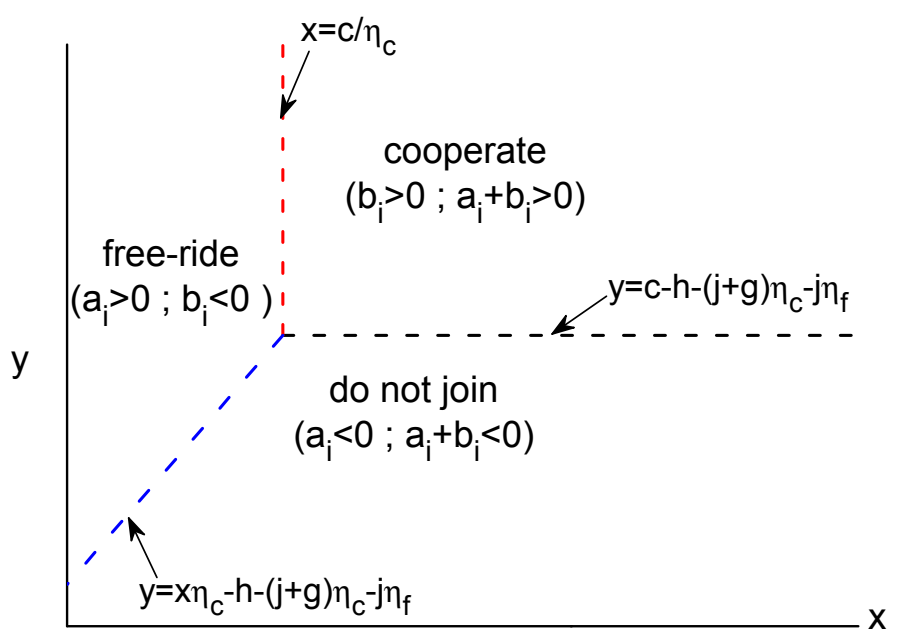

Figure 1: Boundaries between regions corresponding to the different individual choices, as a function of the values of the (dimensionless) quenched random variables $y_{i}$ and $x_{i}$.

In order to get some insight about the problem, it is useful to consider a plane whose axes are the quenched random variables $x$ (abscissas) and $y$ (ordinates), where each individual $i$ is represented by a point according to his values $\left(x_{i}, y_{i}\right)$. Then, for any given values of $\eta_{c}$ and $\eta_{f}$, the lines

$$
\begin{aligned}
y & =y_{m} \equiv c-h-(j+g) \eta_{c}-j \eta_{f}, \\
y & =y_{m}-c+x \eta_{c}, \\
x & =x_{m} \equiv \frac{c}{\eta_{c}}
\end{aligned}
$$

represent the marginal individuals whose utilities are at the boundaries between different optimal strategies. These lines partition the $(x, y)$ plane into three regions, represented on figure 1. The optimal strategy of individuals whose $\left(x_{i}, y_{i}\right)$ values lie in the upper-right region, defined by

$$
\begin{array}{ll}
x_{i}>x_{m} & \text { or } \quad b_{i}>0, \\
y_{i}>y_{m} & \text { or } \quad a_{i}+b_{i}>0,
\end{array}
$$


is to cooperate. The best strategy for those whose $\left(x_{i}, y_{i}\right)$ satisfy

$$
\begin{array}{rll}
x_{i}<x_{m} & \text { or } & b_{i}<0 \\
y_{i}>y_{m}-c+x_{i} \eta_{c} & \text { or } & a_{i}>0
\end{array}
$$

is to free-ride. Individuals with $\left(x_{i}, y_{i}\right)$ in the lower-right region do not join the community.

\section{Mean field dynamics}

We are interested in the temporal evolution of the system within an iterated game setting, in which individuals have to choose their best strategies $s_{i}(t)$ based on the available information. We assume that each time an agent has to make a decision, he has the exact information of the global proportions of cooperators and free-riders at the preceding outcome, $\eta_{c}(t-1)$ and $\eta_{f}(t-1)$, and uses these quantities to estimate his surplus (2). Such a dynamics is called Cournot best reply in economics literature. In simulations, starting from initial guesses $\eta_{c}(0)$ and $\eta_{f}(0)$, the updating is said to be in parallel if all the individuals in the population first determine their best strategies based on the preceding outcome, and make their decisions simultaneously afterwards. At the opposite, in random sequential updating, a single individual selected at random is asked to make his decision at each time step. The latter dynamics simulates systems where the individuals make their decisions without any temporal correlation. In order to compare the time scales of both dynamics, it is usual to consider that $N$ sequential time steps are equivalent to one parallel update. Intermediate updating schemes may be implemented, but here we only consider these two extreme cases, that are standard in economics and in statistical physics.

Referring back to figure 1, since the boundary lines depend on the values of $\eta_{c}(t)$ and $\eta_{f}(t)$, they will shift in the course of updating according to the perceived proportions of cooperators and free-riders. Individuals whose values of $y_{i}$ and $x_{i}$ lie close to the boundaries are susceptible to small changes of $\eta_{c}$ and $\eta_{f}$ : their strategies may change with time, and in turn induce changes in those of the others.

Hereafter we study how the system reaches its stable states upon successive updates, that is, the path followed by a point representative of the system's state in the plane $\left(\eta_{c}, \eta_{f}\right)$. Such paths should either end up in a stable fixed point or get trapped in other types of attractors if they exist. In the following we consider separately the parallel and sequential updating schemes, since the corresponding dynamic equations are different.

Let us introduce the complementary cumulative functions defined by:

$$
G_{\chi}(\zeta) \equiv 1-F_{\chi}(\zeta)=\int_{\zeta}^{\infty} f_{\chi}(\xi) d \xi
$$


where $\chi \in\{X, Y\}$, and $F_{\chi}(\zeta)$ is the cumulative distribution of $f_{\chi}$. Using the fact that $y$ is independent of $x$, the probabilities $p$ and $q$ that an individual $i$ taken at random will cooperate or free-ride respectively at the next time step given values of $\eta_{c}(t)$ and $\eta_{f}(t)$ are

$$
\begin{aligned}
p\left(s_{i}(t+1)=1\right) & \equiv p\left(\eta_{c}(t), \eta_{f}(t)\right)=\int_{x_{m}(t)}^{\infty} f_{X}(x) d x \int_{y_{m}(t)}^{\infty} f_{Y}(y) d y \\
& =G_{X}\left(x_{m}(t)\right) G_{Y}\left(y_{m}(t)\right), \\
q\left(s_{i}(t+1)=-1\right) & \equiv q\left(\eta_{c}(t), \eta_{f}(t)\right)=\int_{-\infty}^{x_{m}(t)} \int_{y_{m}(t)-c+x \eta_{c}(t)}^{\infty} f_{X}(x) f_{Y}(y) d y d x \\
& =\int_{0}^{x_{m}(t)} f_{X}(x) G_{Y}\left(y_{m}(t)-c+x \eta_{c}(t)\right) d x
\end{aligned}
$$

where $y_{m}(t)$ and $x_{m}(t)$ are given by equations (9c) and (9a) respectively with $\eta_{c}=\eta_{c}(t)$ and $\eta_{f}=\eta_{f}(t)$.

\subsection{Fixed point equations}

We consider the limit of very large populations, namely $N \rightarrow \infty$, for which $\eta_{c}$ and $\eta_{f}$ are unbiased estimators of the probabilities of cooperate and freeride respectively. Since at a fixed point the probabilities of the possible strategies are stationary, they satisfy equations (13) with $p=\eta_{c}, q=\eta_{f}$ :

$$
\begin{aligned}
\eta_{c} & =p\left(\eta_{c}, \eta_{f}\right), \\
\eta_{f} & =q\left(\eta_{c}, \eta_{f}\right) .
\end{aligned}
$$

Notice that the trivial solution $\eta_{c}=0$ satisfies (14a), corresponding to a state where nobody contributes to the public good. The community survives because when $\eta_{c}=0$ free-riders do not suffer from any moral burden. The model in this case reduces to the model with (symmetric) social interactions $j$ considered in [16]. Here $\eta_{f}$ plays the role of the fraction of customers, and may present a single or two fixed points depending on whether the interaction strength $j$ is smaller or larger, respectively, than a critical value $j_{B}=1 / f_{Y}\left(y_{\max }\right)$ where $y_{\max }$ is the maximum of $f_{Y}$, the IWJ distribution.

Notice however that Nash equilibria without cooperators are a drawback of our keeping the model simple enough to be studied analytically. The main question, namely whether polymorphic organizations with coexistence of cooperators and free-riders are stable, is meaningful outside the region $\eta_{c}=0$.

\subsection{Parallel dynamics equations: two-dimensional map}

In parallel dynamics, one assumes that the agents update their strategies simultaneously, starting from an arbitrary initial configuration. The perceived $\eta_{c}$ and $\eta_{f}$ used by all the agents to estimate their utilities are the 
same. The boundary lines between the three regions of figure 1, which depend on $\eta_{c}(t)$ and $\eta_{f}(t)$, partition the population at time $t$ according to their estimated utilities: all the individuals whose idiosyncratic parameters lie in region I will choose to cooperate, those in region II to free-ride and those in region III not to join. In this way, using equations (13), the dynamics can be formulated as the following two-dimensional deterministic map $\Omega:\left(\eta_{c}^{\prime}, \eta_{f}^{\prime}\right)=\left(p\left(\eta_{c}, \eta_{f}\right), q\left(\eta_{c}, \eta_{f}\right)\right)$ in the simplex $\mathbf{S}:=\left\{\left(\eta_{c}, \eta_{f}\right): \eta_{c} \geq 0, \eta_{f} \geq\right.$ $\left.0, \eta_{c}+\eta_{f} \leq 1\right\}$, namely:

$$
\begin{aligned}
\eta_{c}(t+1) & =p\left(\eta_{c}(t), \eta_{f}(t)\right), \\
\eta_{f}(t+1) & =q\left(\eta_{c}(t), \eta_{f}(t)\right) .
\end{aligned}
$$

Thus, the fixed points (14) are the fixed points of the parallel dynamics. The results presented in this section shed light on their nature and allow to determine if there are other kinds of equilibria.

In two-dimensional maps or flows, a fixed point is called a sink if all the points in its neighborhood converge to it, a source if these points diverge from it and a saddle if in one direction the map or flow converges, while in the other directions it diverges. Together these three types are called hyperbolic, which is the only kind of equilibria possibly encountered in a structurally stable system, i.e. a system stable with respect to small variations of the parameters (in our case $h, j, g, c$ and $d$ ). For our two-dimensional map, the nature of an equilibrium point $\left(\eta_{c 0}, \eta_{f 0}\right)$ is determined by the following Jacobian matrix

$$
\mathbf{J}_{m}=\left[\begin{array}{ll}
\partial p\left(\eta_{c 0}, \eta_{f 0}\right) / \partial \eta_{c} & \partial p\left(\eta_{c 0}, \eta_{f 0}\right) / \partial \eta_{f} \\
\partial q\left(\eta_{c 0}, \eta_{f 0}\right) / \partial \eta_{c} & \partial q\left(\eta_{c 0}, \eta_{f 0}\right) / \partial \eta_{f}
\end{array}\right]
$$

Denoting the two eigenvalues of $\mathbf{J}_{m}$ as $\omega_{1}$ and $\omega_{2}$, then the fixed point is a sink if $\left|\omega_{1}\right|<1$ and $\left|\omega_{2}\right|<1$, a source if $\left|\omega_{1}\right|>1$ and $\left|\omega_{2}\right|>1$ and a saddle if $\left(\left|\omega_{1}\right|-1\right)\left(\left|\omega_{2}\right|-1\right)<0([17] \S 1.4)$. There are two types of curves emanating from each saddle point $\mathbf{x}_{0}$, namely the stable manifold, defined as

$$
W_{s}\left(\mathbf{x}_{0}\right)=\left\{x \in \mathbb{R}^{2} \mid \Omega^{t}(\mathbf{x}) \rightarrow \mathbf{x}_{0} \text { as } t \rightarrow \infty\right\}
$$

and the unstable manifold, defined as

$$
W_{u}\left(\mathbf{x}_{0}\right)=\left\{x \in \mathbb{R}^{2} \mid \Omega^{-t}(x) \rightarrow \mathbf{x}_{0} \text { as } t \rightarrow \infty\right\}
$$

$\Omega^{-1}$ denoting a backward iterate of the map. The boundaries of the basins of attraction are usually formed by stable manifolds. Moreover, if a transversal intersection between the stable and unstable manifolds of the same saddle point exists, the map will exhibit chaotic behavior. 


\subsection{Random sequential dynamics equations: two-dimensional flow}

In sequential dynamics, one updates one agent chosen at random in each time step. It is usual to consider $N$ (the number of agents in the system) successive updates (called one Monte-Carlo step) as being comparable to one step of parallel updating, i.e., the time step between two successive individual updatings is $\tau=1 / N$. is

In each individual update, the expected displacement of the pair $\left(\eta_{c}, \eta_{f}\right)$

$$
\begin{aligned}
\eta_{c}(t+\tau) & =\eta_{c}(t)+(1 / N)\left(p\left(\eta_{c}(t), \eta_{f}(t)\right)-\eta_{c}(t)\right), \\
\eta_{f}(t+\tau) & =\eta_{f}(t)+(1 / N)\left(q\left(\eta_{c}(t), \eta_{f}(t)\right)-\eta_{f}(t)\right),
\end{aligned}
$$

where $p$ and $q$ are defined in (13), and the factor $1 / N$ is the probability of selecting the agent that is updated. Taking the continuous limit $N \rightarrow \infty$, we have the following set of differential equations:

$$
\begin{aligned}
\frac{d \eta_{c}}{d t} & =p\left(\eta_{c}, \eta_{f}\right)-\eta_{c} \\
\frac{d \eta_{f}}{d t} & =q\left(\eta_{c}, \eta_{f}\right)-\eta_{f}
\end{aligned}
$$

with the time unit being one Monte-Carlo step. Now the system evolves as an autonomous system in a planar phase space, i.e. as a two-dimensional flow in $\mathbf{S}$. Clearly, the fixed points of the sequential dynamics are the same as the equilibria (14).

The three generic types of equilibria, namely source, sink and saddle exist in a structurally stable two-dimensional flow as well. The nature of an equilibrium point $\left(\eta_{c 0}, \eta_{f 0}\right)$ is now determined by the Jacobian matrix of the flow

$$
\mathbf{J}_{f}=\left[\begin{array}{cc}
\partial p\left(\eta_{c 0}, \eta_{f 0}\right) / \partial \eta_{c}-1 & \partial p\left(\eta_{c 0}, \eta_{f 0}\right) / \partial \eta_{f} \\
\partial q\left(\eta_{c 0}, \eta_{f 0}\right) / \partial \eta_{c} & \partial q\left(\eta_{c 0}, \eta_{f 0}\right) / \partial \eta_{f}-1
\end{array}\right]
$$

Denoting the eigenvalues of $\mathbf{J}_{f}$ as $\omega_{1}^{\prime}$ and $\omega_{2}^{\prime}$, then the point is a sink if $\omega_{1}^{\prime}<0$ and $\omega_{2}^{\prime}<0$, a source if $\omega_{1}^{\prime}>0$ and $\omega_{2}^{\prime}>0$ and a saddle if $\omega_{1}^{\prime} \omega_{2}^{\prime}<0$ ([17] $\S 1.2 \sim 1.3)$. Note that the condition for an equilibrium to be a saddle gives the same inequality as in the parallel case. Therefore, the set of saddle points in sequential updating coincides with that in parallel updating. However, the set of sinks or sources are not necessarily the same in both cases, since the governing inequalities are quite different. The definitions of the stable and unstable manifolds are analog to those in the two-dimensional map with the discrete time step replaced by the continuous time variable $t$. According to the Poincaré-Bendixson Theorem ([17] Theorem 1.8.1), the two-dimensional flow system will never go into chaos. 


\section{Equilibria for particular distributions $f_{X}$ and $f_{Y}$}

To go further with the analysis of the preceding section we need to specify the pdfs of the idiosyncratic parameters. In this paper we present the most interesting of the cases we have studied, in which the $x_{i}$ follow a uniform distribution of finite width $d$ :

$$
\begin{aligned}
& f_{X}(x)=\frac{1}{d} \text { for } 0 \leq x \leq d, \\
& f_{X}(x)=0 \text { otherwise. }
\end{aligned}
$$

and the $y_{i}$ are distributed according to:

$$
f_{Y}(y)=\frac{1}{4 \cosh ^{2}(y / 2)} .
$$

The cumulative function corresponding to (21) is the logistic distribution. The complementary functions (12) are:

$$
\begin{aligned}
& G_{X}(z)=(1-z) \Theta(1-z), \\
& G_{Y}(z)=1 /[1+\exp (z)],
\end{aligned}
$$

where $\Theta$ is the Heaviside function.

\section{Fixed points}

The fixed point equations (14) may be written as follows:

$$
\begin{aligned}
\eta_{c} & =\left(1-\frac{\rho}{\eta_{c}}\right) \Theta\left(1-\frac{\rho}{\eta_{c}}\right) G_{Y}(c-Z) \\
\eta_{f} & =\frac{\rho}{c \eta_{c}} \log \left[1+\left(e^{c}-1\right) G_{Y}(c-Z)\right],
\end{aligned}
$$

where $\rho \equiv c / d$ and $Z \equiv h+j\left(\eta_{c}+\eta_{f}\right)+g \eta_{c}$. Since $f_{X}$ has a bounded support, equation (23a) vanishes if $0 \leq \eta_{c}(t) \leq \rho$. Thus, if $\eta_{c}(0) \leq \rho$, the system evolves towards the trivial equilibrium without cooperators. In parallel dynamics, equation ( $15 \mathrm{a}$ ) gives $\eta_{c}(1)=0$ : the state with no cooperators is reached after a single parallel update. Afterwards, on the axis $\eta_{c}=0, \eta_{f}$ evolves according to equation (15b),

$$
\eta_{f}(t+1)=G_{Y}\left(-h-j \eta_{f}(t)\right) .
$$

The equilibrium value of $\eta_{f}$ is given by (14b), obtained by replacing $\eta_{f}(t)$ and $\eta_{f}(t+1)$ by $\eta_{f}$ in the above equation. As already mentioned (see [16]) there is a critical value of $j, j_{B}$, such that for $j<j_{B}$, there is a single equilibrium. In that case all the points $\eta_{c}(0)<\rho$ will eventually be mapped to it. If $j>j_{B}(14 \mathrm{~b})$ has three solutions. One of them, $\eta_{f u}$, is an unstable fixed point separating the basin of attraction of the two others, 
that are stable. To summarize, when $\eta_{c}(0)<\rho$, after a first time step the system is mapped to the axis $\eta_{c}=0$ and then evolves, following (24), either to one fixed point (if $j<j_{B}$ ) or to one of two fixed points (if $j>$ $\left.j_{B}\right)$ depending on whether $\eta_{f}(0)>\eta_{f u}$ or $\eta_{f}(0)<\eta_{f u}$. In the case of the logistic distribution $f_{Y}$ considered here, $j_{B}=4$ [15]. Under random sequential updating, since $p\left(\eta_{c}(0), \eta_{f}(0)\right)=0$ for $0 \leq \eta_{c}(0) \leq \rho$, equation (19a) shows that $\eta_{c}$ vanishes exponentially fast, the fixed points being the same as for the parallel dynamics. These solutions have been described here for completeness since, as already stated, they are not within the scope of the questions addressed by the model

Notice that there are no fixed points with $\eta_{f}=0$ : since $G_{Y} \geq 0$, the right hand side of (23b) vanishes only if $c=0$, i.e. full cooperation may exist only if cooperation is costless.

When $\eta_{c}(0)>\rho$, calling $G$ the value taken by $G_{Y}(c-Z)$, equation (23a) gives $\eta_{c}=\left(1-\frac{\rho}{\eta_{c}}\right) G$, which can be solved for non-vanishing $\eta_{c}$ in terms of $G$ :

$$
\eta_{c}=\eta_{c}^{ \pm}[G] \equiv \frac{1}{2} G\left\{1 \pm\left[1-\frac{4 \rho}{G}\right]^{1 / 2}\right\}
$$

Later we shall see that both the + and the - branches may give stable equilibria, though the parameter range for which a stable equilibrium exists for the - branch is much narrower than for the + branch. From (25) one gets that the Nash equilibria with cooperators satisfy $\eta_{c} \geq 2 \rho$ (equality can occur when $j=g=0$ ): the fraction of cooperators is larger than a threshold given by the cooperation cost relative to the average weight $(\bar{x}=d / 2)$ of the social disapproval for free-riding.

Equation (23b) can also be parameterized in terms of $G$ by defining

$$
\eta \equiv \frac{j\left(\eta_{c}+\eta_{f}\right)+g \eta_{c}}{j+g}
$$

Introducing (25) and (23b) into (26) we obtain an equation for $\eta$ :

$$
\eta=\eta_{1}[G] \equiv \eta_{c}^{ \pm}[G]+\frac{j}{j+g} \frac{1}{\eta_{c}^{ \pm}[G]} \frac{1}{d} \log \left[1+\left(e^{c}-1\right) G\right]
$$

Inverting (22b) for $z=c-h-(j+g) \eta$, we obtain $\eta$ in terms of $G$ :

$$
\eta=\eta_{2}[G] \equiv \frac{1}{j+g}\left\{c-h-\log \frac{1-G}{G}\right\} .
$$

The possible solutions $\eta$ are then obtained by the intersects of the curves $\eta_{1}[G]$ and $\eta_{2}[G]$. Introducing the corresponding value of $G$ into (25) allows to determine $\eta_{c}$, and then $\eta_{f}$ is deduced by introducing the values of $\eta_{c}$ and $\eta$ into (26). An example of curves $\eta_{1}[G]$ and $\eta_{2}[G](G \in[0,1])$ is shown on Figure (2). There are up to 3 intersects (for $h-c=-3.3$ ), although one can check that at most 2 correspond to stable fixed points. 


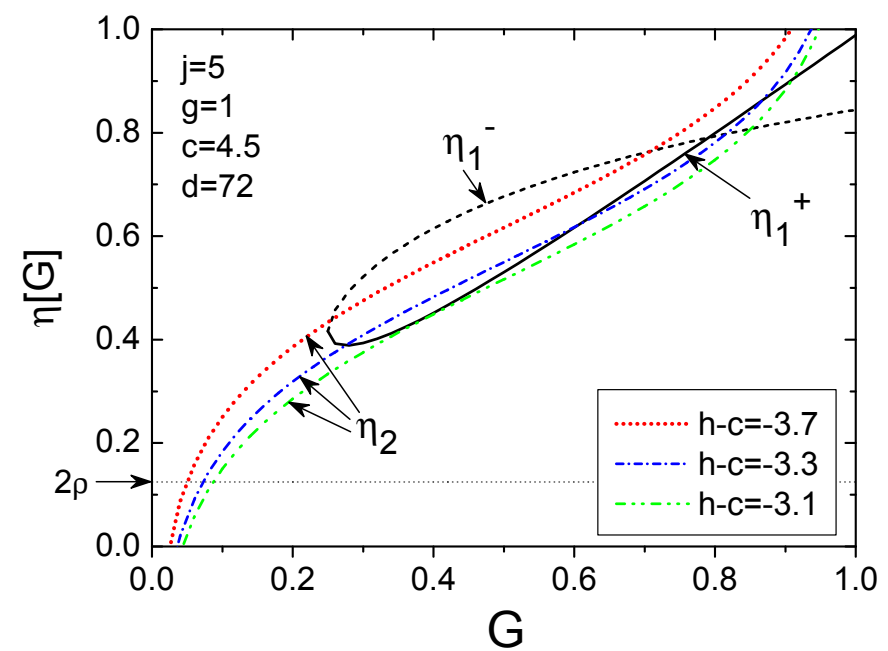

Figure 2: Fixed points. Solution by curve intersection for distributions $f_{X}$ and $f_{Y}$ given by equations (20) and (21).

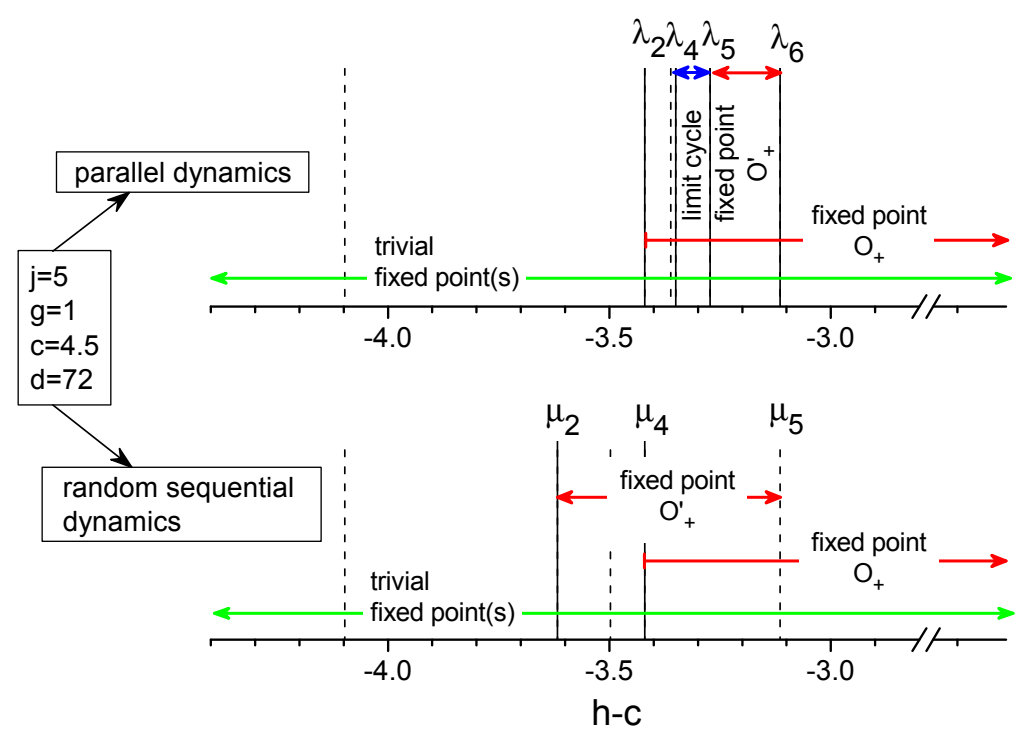

Figure 3: Regions of existence of the different stable equilibria under parallel and random sequential dynamics (see appendices A.1 and A.2 for the values of the $\lambda_{n}$ and the $\left.\mu_{n}\right)$. 


\section{Equilibria with parallel dynamics.}

Notice that the above analysis allows to determine the fixed points of the system, but doesn't give any hint about the existence of cycles. We have thus numerically computed the stable and unstable manifolds for each saddle point, with the same set of values for $j, g, c$ and $d$ as in figure 2, while changing $h-c$ from -4 to -3 in steps of 0.02 . We summarize here the main results, leaving the details to appendix A.1.

Besides the trivial fixed point(s) with $\eta_{c}=0$, for any $h-c \geq-3.4201$ there is a fixed point $O_{+}$corresponding to a relatively large organization having a proportion of cooperators larger than the proportion of free-riders. This occurs for cooperation costs relatively large compared to the average willingness to join ( $h-c$ is negative). The value of $j$ considered is large enough to compensate negative values of $h_{i}-c$ on a large fraction of the population, producing a bandwagon effect. For $-3.3504<h-c<-3.1146$, this fixed point $O_{+}$coexists with another non-trivial attractors (see figure 3 ). For $-3.3504<h-c<-3.2726$, the attractor is a limit cycle $C_{S}$. It shrinks (at $h-c=-3.2726$ ) to a second fixed point $O_{+}^{\prime}$ which corresponds to an organization with qualitatively similar proportions of cooperators and free riders, and with fewer members than at the equilibrium $O_{+}$. This second fixed point $O_{+}^{\prime}$ exists for $-3.2726<h-c<-3.1146$. For larger values $(h-c>-3.1146)$ it disappears, leaving only the fixed point $O_{+}$with a very large basin of attraction.

Figures 4 and 5 present the results for two values of $h-c$ in the regions where we expect coexistence of the two non-trivial attractors. On the left hand side are represented the different manifolds predicted by the mean field theory (details lefts to Appendix A.1). On the right hand side we present the dynamical paths obtained under parallel dynamics updating of a system with $N=1000$ agents with the same parameters, starting from different initial conditions. The agreement with the analytical results is very good, although there are some finite size effects (see the figure's legend). One of the most striking result of the simulations are the winding trajectories that turn around over long times before reaching the corresponding attractor. This means that an oscillatory behavior might be observed in actual systems even before convergence to the cycle attractor. Notice also that these oscillations may exist even if the system is expected to reach a Nash equilibrium.

\subsection{Equilibria with random sequential dynamics}

We have also computed the stable and unstable manifolds for the flow, the mean field approximation for sequential dynamics. The results, presented in appendix A.2 are summarized on figure 3. They are similar to those with parallel dynamics, although for the parameters considered $(j=5, g=1$, $c=4.5$ and $d=72$ ) the system only presents fixed points. Cycles exist, but 

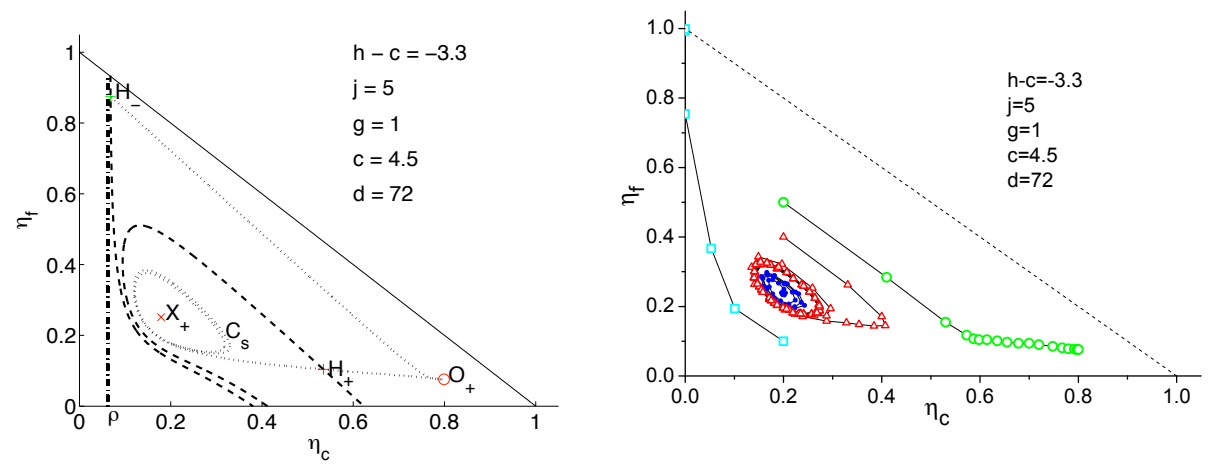

Figure 4: Left. Stable and unstable manifolds of the theoretical saddle points in the plane $\left(\eta_{c}, \eta_{f}\right)$ for the same parameter values as in figure 3 , with $h-c=-3.3$. The non trivial attractors are a stable cycle $C_{s}$ (its basin of attraction is limited by the dashed line corresponding to the stable manifold of $H_{+}$, with its two ends at the line $\left.\eta_{f}=0\right)$. The point $O_{+}$is a fixed point. Its basin of attraction is the region bounded by the stable manifold of $H_{-}$between the lines $\eta_{c}+\eta_{f}=1$ and $\eta_{f}=0$, excluding the basin of attraction of $C_{s}$.

Right. Trajectories of the simulated system for four different initial conditions, that converge respectively to a trivial fixed point (squares), to a cycle of length 41 (empty triangles), to a fixed point (full circles) not predicted by the mean field equations (due to finite size effects) and to the non-trivial fixed point $O_{+}$(empty circles).
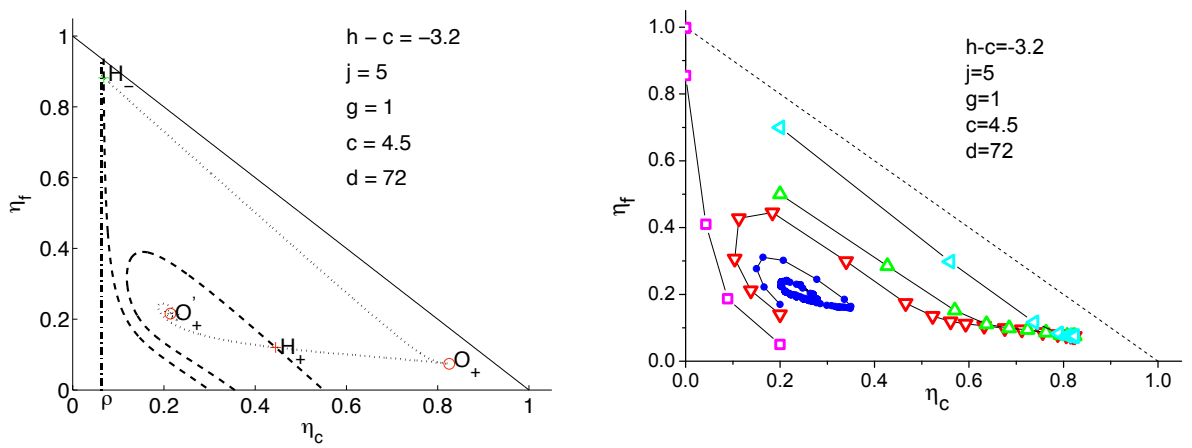

Figure 5: Left. Theoretical equilibria for the map in the plane $\left(\eta_{c}, \eta_{f}\right)$, for the same parameter values as in figure 3 , with $h-c=-3.2$. The stable limit cycle $C_{s}$ has merged with the source $X_{+}$through a Hopf bifurcation to become a sink $O_{+}^{\prime}$, whose basin of attraction is the stable manifold of the saddle $H_{+}$.

Right. Trajectories of the simulated system (parallel dynamics) for five different initial conditions. One of them converges to a trivial fixed point (squares), one (circles) to a cycle of length 13 (instead of the predicted non-trivial fixed point $O_{+}^{\prime}$ ), the three others (triangles) converge to the non-trivial fixed point $O_{+}$, which has a large basin of attraction. 
for other parameter values, as discussed in the appendix.

Figure 6 presents an example of evolution of the same system under parallel and sequential dynamics, showing the dramatic difference between both types of updating rules.

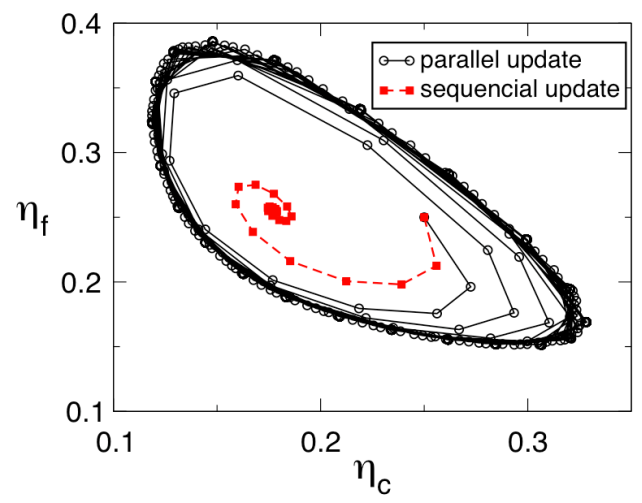

Figure 6: Examples of trajectories for parallel and random sequential dynamics starting from the same initial conditions, for a system with the same parameter values as in figure 3 , and $h-c=-3.3$.

\section{$5 \quad$ Simulations results and phase diagrams}

The theoretical results of the preceding sections predict the behavior of an infinite system. On figure 7 we present the phase portrait obtained through parallel dynamics with systems of different sizes, for $h-c=-3.3$ and the same set of parameters as in figure 4. Starting from different initial conditions, we determined the attractors in the phase space $\left(\eta_{c}, \eta_{f}\right)$. As $N$ decreases, more and more fixed points invade the region where cycles exist, and many different cycles appear. This multiplicity of attractors is due to missing values of the IWJ in the finite population. However, the mean field predictions, and in particular the existence of cycles, remain qualitatively correct.

In order to get deeper insight on the phase diagram of the model, we studied the attractors of large systems as a function of the different parameters. Results as a function of $h-c$, summarized on figure 8 , are in very good agreement with the mean field predictions, as may be seen through the position of the theoretical boundaries $\lambda_{n}$.

Figures 9 present separately the $\eta_{c}$ values at the fixed points and at the cycles for systems of different sizes, ranging from $N=1000$ to $N=100000$, as a function of $h-c$. Consistently with the above results, the size effects are important, but do not modify qualitatively the phase diagram.

As may be seen on figure 10 (left), the fraction of the phase space that 


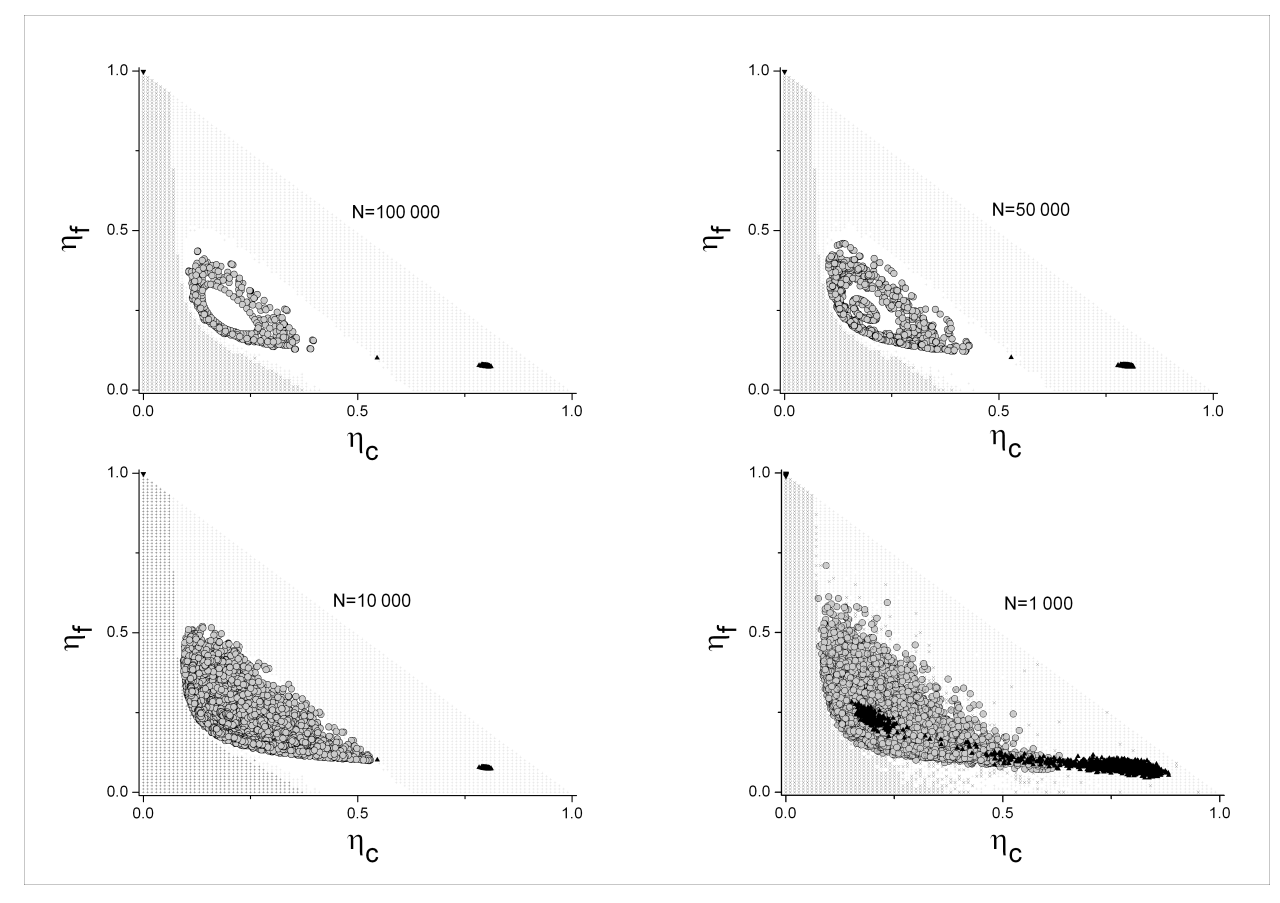

Figure 7: Attractors and their basins of attraction for different system sizes, obtained by starting from all the possible initial conditions (with a mesh of 0.01). Parameters are: $j=5, g=1, d=72, h=1.2$. The white region is the basin of attraction of the cycles.

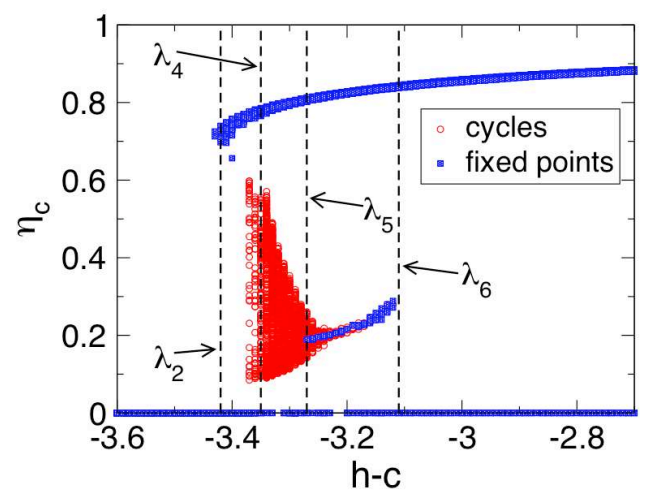

Figure 8: Attractors for parallel dynamics with $N=100000$, for 20 simulated systems, with the same parameters as in the preceding figures, i.e. $j=5, g=1$, $c=4.5$ and $d=72$. The points in this and the following figures are obtained as follows: we draw 20 systems at random with probabilities $f_{X}$ and $f_{Y}$. The points in the figures correspond to the final states obtained for each system, starting from each possible initial point in the plane $\eta_{c}, \eta_{f}$ laying on a grid of mesh 0.1 satisfying the condition $\eta_{c}+\eta_{f} \leq 1$. That corresponds to 45 different initial conditions for each of the 20 systems, i.e. a total of 900 simulations. 

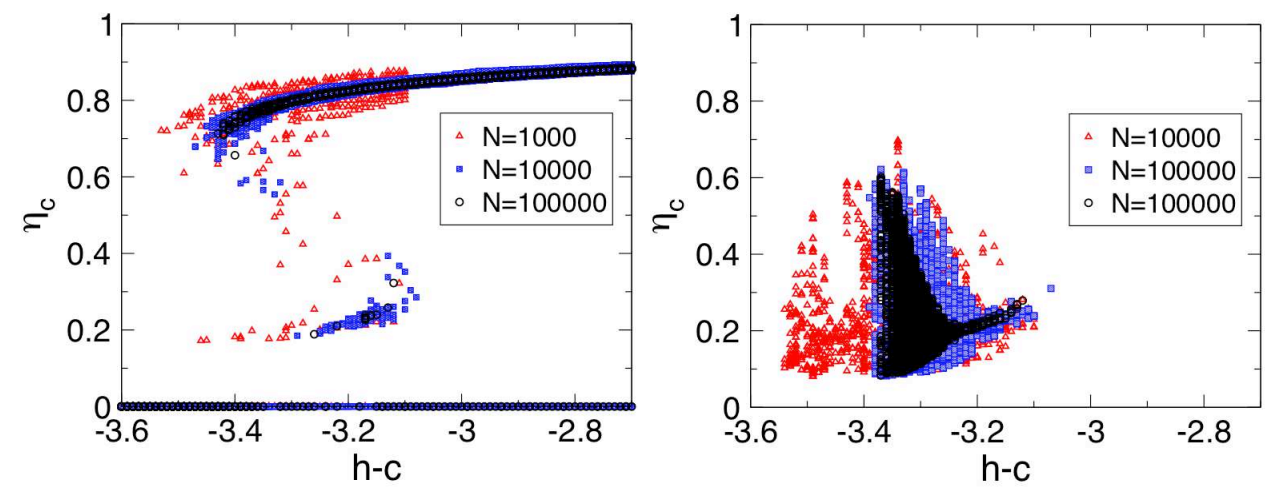

Figure 9: Fixed points (left) and cycles (right) obtained through simulations of systems of different sizes.

corresponds to cycles increases dramatically with the width of the $x_{i}$ distribution, as expected, since larger values of $d$ correspond to higher anisotropy in the interactions. The attractors as a function of the strength $j$ of the social interactions is represented on figure 10 (right). We observe that when the bandwagon effect is large enough, the cycles disappear and only the fixed point $O_{+}$with a large fraction of cooperators survives (besides the trivial fixed point).
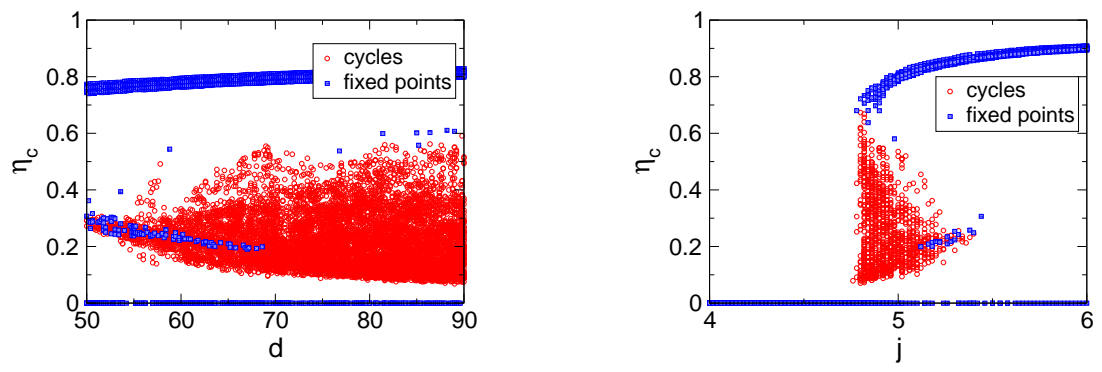

Figure 10: Phase diagram: Attractors as a function of $d$ (left) and of $j$ (right).

\section{Discussion}

In order to understand the existence of the limit cycles, we analyzed in details the dynamics of a particular system with $N=100$ individuals. We isolated on figure 11 the agents involved in the limit cycle, identified by the values of their parameters $x_{i}$ and $y_{i}$. As expected, these values are close to the marginal values (9), i.e. they lie close to the phase boundaries defined 
in figure 1. Notice that these boundaries depend on the values of $\eta_{c}$ and $\eta_{f}$, so that their positions change on time. In the course of the cycle, some individuals oscillate between states $s_{i}=1$ and $s_{i}=0$, others between $s_{i}=1$ and $s_{i}=-1$, and others between $s_{i}=-1$ and $s_{i}=0$. Some even go successively through the three possible states, as may be seen in the inset of figure 11 .

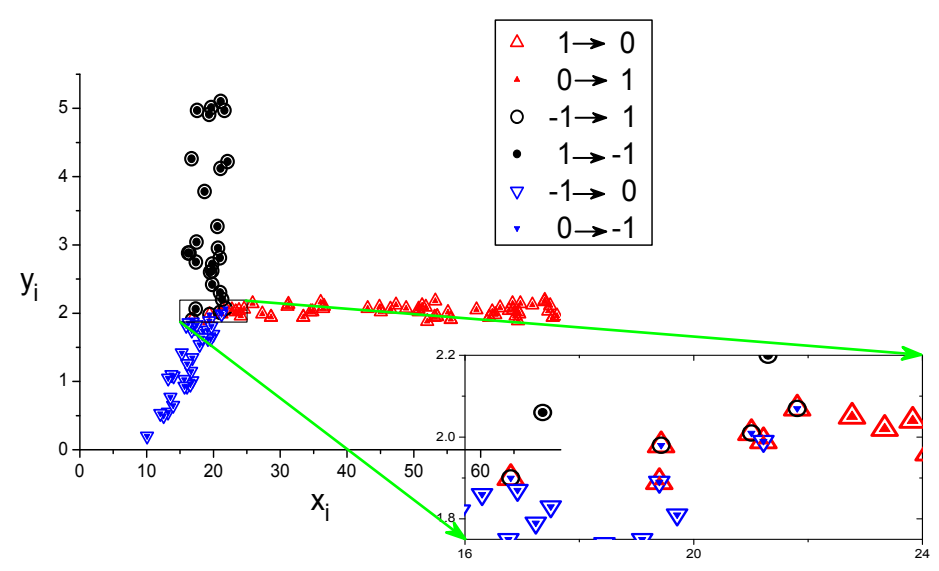

Figure 11: Parameters $(x, y)$ space. Individuals involved in a cycle: in red those that oscillate between cooperating or not to participate at all (outsiders), in black those that oscillate between cooperating or free-riding, and in blue those that either free-ride or do not participate. Notice (see inset) that some individuals may adopt alternatively each of the three possible states.

Intuitively, the dynamics of the cycle is as follows: assume that at some instant $t$ (like at $t=26$ in figure 12) the system has proportions $\eta_{c}(t)$ and $\eta_{f}(t)$. Then, as more cooperators enter the organization, the cost of freeriding becomes too high for some agents in the organization (those with relatively large $x_{i}$ ). These will leave the organization, decreasing thus the total number of members. As a consequence, the value of the organization decreases for everybody and both the fractions of cooperators and of free-riders at the following periods will decrease. At some point, certain individuals may join the organization as free-riders, because the social pressure due to cooperators has decreased. As a result, the total number of members increases making it worth for some cooperators to join the organization, initiating a new oscillation. As may be seen on figure 12, the cycle is actually closed (in the mathematical sense) only after many oscillations, when the fractions of cooperators and free-riders repeat themselves exactly. It is interesting that the total fraction of members also oscillates, but with a different phase, revealing the complexity of the dynamics. 


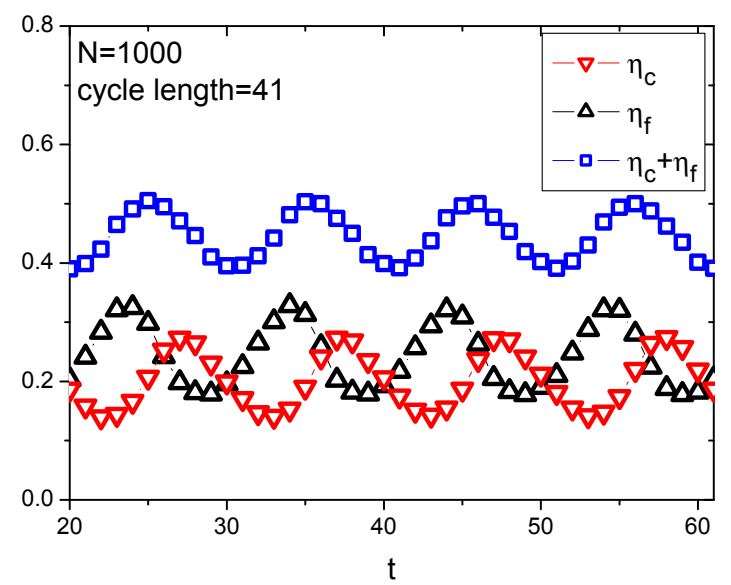

Figure 12: Cycles. Composition of the system for $N=1000$.

In the computer simulations, the length of the cycles depend very strongly on the particular realization of the random variables $y_{i}$ and $x_{i}$. Figures 13 show examples for different system sizes. Notice that the length of the cycles depend on the system's details (the particular realization of the random variables $x_{i}$ and $y_{i}$ ) even for systems with 1 million agents. Finally, let us point out that our results remain qualitatively unchanged if $G=0$. In contrast, the existence of cycles requires $J>0$, as well as the two idiosyncratic components, namely, the willingness to join $H_{i}$ and the moral burden weight $X_{i}$. In fact, it is straightforward to show that for $J=0$ the dynamics converges necessarily to fixed points.

\section{Conclusion}

In this paper we studied in details a model of a charity organization, or any other organization whose value is proportional to the number of its members [18]. Individuals may not participate, and those joining the organization may or not cooperate. Thus, the size of the organization is an emergent property, not fixed a priori.

We investigated whether such a voluntary organization may survive, and under what conditions. Similar situations have been studied within the public goods literature. According to the standard game theoretical assumption of selfish individuals, such an organization is expected to be exclusively composed of free-riders. However, daily life experience and experimental economics results have shown that some level of cooperation often exists, a fact attributed to moral feelings. 

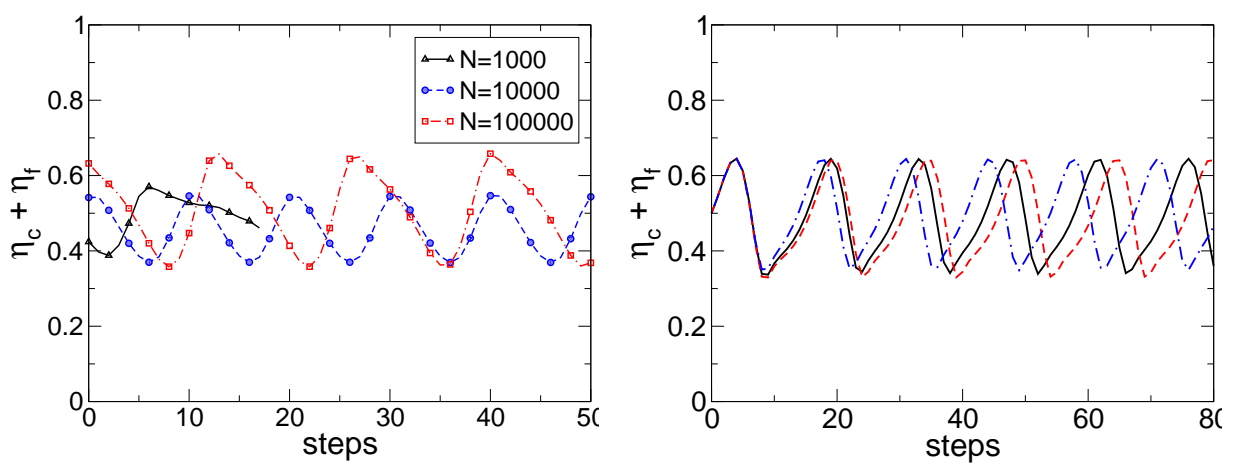

Figure 13: Cycles. Total fraction of community members as a function of $t$ for different system sizes (left) and for three different systems with $N=10^{6}$.

The model considers heterogeneous individuals; they have idiosyncratic preferences for joining the community and they also weight idiosyncratically the social disapproval for free-riding. The surplus of the community members is proportional to their number, be they cooperators or not, but cooperators contribute more than free riders to the community value. Upon joining, cooperators bear a fixed cost while free-riders suffer a cost due to cooperators' disapproval, that they weight idiosyncratically.

We have shown that in this model there are three kinds of equilibrium attractors. There is a trivial Nash equilibrium for all the parameters of the model, in which the organization is exclusively composed of free-riders. Thus, the population is partitioned into two categories: the outsiders (those that do not join the community) and the free-riders. Such an equilibrium between only two strategies exists because in the model the community has a value for its members even if nobody cooperates. In this extreme case there is no cost for free-riding, and the parameter $X_{i}$ does not play any role. The system's properties are the same as those of simple bandwagon models where the individuals have to make a binary choice (to buy or not a good at a given price, participate or not to a riot, etc). It has been shown [16] that for some values of the pertinent parameters $(h-$ the population average willingness to join-, and $j$ - the strength of the bandwagon effect -), there may be one or two fixed points. Coexistence of two possible collective states 
(Nash equilibria), having either a large or a small fraction of free-riders, arise for values of $h$ smaller than, and $j$ larger than, threshold values that depend on the distribution of the idiosyncratic component $H_{i}$. Besides this trivial equilibrium, the model considered here presents one or two Nash equilibria with finite fractions of cooperators and free-riders.

An interesting result of this model is that besides the Nash equilibria, it exhibits limit cycles with corresponding fractions of cooperators, of free-riders (and their sum - the fraction of the population that joins the organization-) oscillating through time. This phenomenon has its root in the strong asymmetry of the interaction between agents, introduced by the idiosyncratic weights that free-riders attach to social disapproval. Although we studied in details the case where these weights $X_{i}$ are uniformly distributed, we verified that the same kind of oscillations exist in the case of a bimodal distribution ${ }^{2}$. The fixed points in this case may be found analytically.

We studied in details the dynamics of the cycles under parallel and random sequential updating both analytically and numerically. Assuming a global neighborhood (i.e. every agent's surplus depends on the fractions of cooperators and free-riders in the whole population), we obtained results which are exact in the asymptotic limit $N \rightarrow \infty$. The two-dimensional dynamical maps and flows have been studied for a large range of parameters. Although qualitatively similar, the phase diagrams of maps and flows corresponding to the same parameters are not identical. The fact that a rich class of bifurcations can occur within a narrow parameter region is in itself truly remarkable. A natural question to ask is what happens in intermediate regimes, where subsets of agents make decisions simultaneously, while others make them at independent times.

Numerical simulations of finite size systems with parallel dynamics qualitatively agree with the analytical results. The dynamical behaviour of the simulated systems show winding trajectories in very large regions of the phase diagram. Oscillations in the fraction of cooperators and free-riders are thus expected in such systems before the onset of the cycle and even when the attractor is a fixed point. We are currently performing simulations with sequential dynamics, where the notion of cycle is not well defined, due to the randomness in the order in which the agents' decisions are updated. Preliminary results also exhibit the above mentionned oscillatory behavior, but the analysis of the data is much more cumbersome.

Future work should focus on the dynamics for interactions restricted to closer neighborhoods, which probably requires more sophisticated techniques from the theory of dynamical systems. Another interesting question

\footnotetext{
${ }^{2}$ Note added in proof: A similar model for an asset market with two kinds of agents, that corresponds to a bimodal distribution of the $X_{i}$, restricted to binary decisions [19] was shown to present cycles.
} 
is how the phase diagram is modified in the case where the agents learn from past actions and can form expectancies.

\section{Acknowledgements}

M.B.G. and J-P.N. are members of CNRS. S.G. thanks support from FAPERGS and $\mathrm{CNPq}$ (Brazil) and also from CNRS during his stay in Grenoble.

\section{References}

[1] Mancur Olson. The Logic of Collective Action : Public Goods and the Theory of Groups. Harvard University Press, 1965.

[2] John O. Ledyard. Public goods: A survey of experimental research. In Roth and Kagel, editors, The Handbook of Experimental Economics, pages 111-194. Princeton University Press, 1995.

[3] E. Fehr and S. Gaechter. Altruistic punishment in humans. Nature, 415:137-140, (2002).

[4] Ernst Fehr and Urs Fischbacher. Social norms and human cooperation. Trends in Cognitive Sciences, 8(4):185-190, 2004.

[5] S. Gaechter and E. Fehr. Collective action as a social exchange. Journal of Economic Behavior and Organization, 39:341-369, (1999).

[6] W. Hichri and A. Kirman. The emergence of coordination in public good games. The European Physical Journal B, 55:149-159, 2007.

[7] Ben Greiner and M. Vittoria Levati. Indirect reciprocity in cyclical networks. an experimental study. Journal of Economic Psychology, 26:711-731, 2005.

[8] Herbert Gintis. Game Theory Evolving. Princeton University Press, 2000.

[9] B. Huberman and N. Glance. Beliefs and cooperation. In Peter Danielson, editor, Modelling Rational and Moral Agents, pages 210-235. Oxford University PressUniversity Press, (1996).

[10] L. A. Imhof, D. Fudenberg, and M. A. Nowak. Evolutionary cycles of cooperation and defection. PNAS, 102:10797-10800, (2005).

[11] G. Iori and V. Koulovassilopoulos. Patterns of consumption in a discrete choice model with asymmetric interactions. In W. Barnett, Ch. Deissenberg, and G. Feichtinger, editors, Economic Complexity: Nonlinear Dynamics, Multi-agents Economies, and Learning, page ISETE Vol 14. Elsevier, Amsterdam, (2004). 
[12] S. N. Durlauf. Statistical mechanics approaches to socioeconomic behavior. In B. Arthur, S. N. Durlauf, and D. Lane, editors, The Economy as an Evolving Complex System II, pages 81-104. Santa Fe Institute Studies in the Sciences of Complexity, Volume XVII, Addison-Wesley Pub. Co, (1997).

[13] M. B. Gordon, D. Phan, R. Waldeck, and J.-P. Nadal. Cooperation and free-riding with moral cost. In Kokinov Boicho, editor, Advances in Cognitive Economics, Proceedings of International Conference on Cognitive Economics (ICCE), Sofia, Bulgaria, August 5-8, pages 294304. Sofia, NBU Press, (2005).

[14] S. N. Durlauf. How can statistical mechanics contribute to social science? Proceedings of the National Academy of Sciences, 96:1058210584, (1999).

[15] J.-P. Nadal, D. Phan, M. B. Gordon, and J. Vannimenus. Multiple equilibria in a monopoly market with heterogeneous agents and externalities. Quantitative Finance, 5(6):557-568, (2006). Presented at the 8th Annual Workshop on Economics with Heterogeneous Interacting Agents (WEHIA 2003).

[16] Mirta B. Gordon, Jean-Pierre Nadal, Denis Phan, and Viktoriya Semeshenko. Discrete choices under social influence: Generic properties. Mathematical Models and Methods in Applied Sciences (M3AS), 2009. Working paper (2007).

[17] J. Guckenheimer and P. Holmes. Nonlinear oscillations, dynamical systems, and bifurcations of vector fields. Springer Verlag, 1990.

[18] Denis Phan, Roger Waldeck, Mirta B. Gordon, and Jean-Pierre Nadal. Adoption and cooperation in communities: mixed equilibrium in polymorphic populations. In Annual Workshop on Economics with Heterogeneous Interacting Agents - WEHIA 2005, June 13-15, University of Essex, UK, (2005).

[19] V. Belitsky, A. L. Pereira, and F. P. de A. Prado. Stability analysis with applications of a two-dimensional dynamical system arising from a stochastic model for an asset market. Presented at ECCS08 (Jerusalem, november 2008), 2008.

[20] J. P. England, B. Krauskopf, and H. M. Osinga. Computing onedimensional stable manifolds and stable sets of planar maps without the inverse. SIAM J. APPLIED DYNAMICAL SYSTEMS, 3(2):161190, 2004.

[21] K. Alligood, T. Sauer, and J.A. Yorke. Chaos: An Introduction to Dynamical Systems. Springer Verlag, 1997. 


\section{A Appendix}

\section{A.1 Phase structure of the maps}
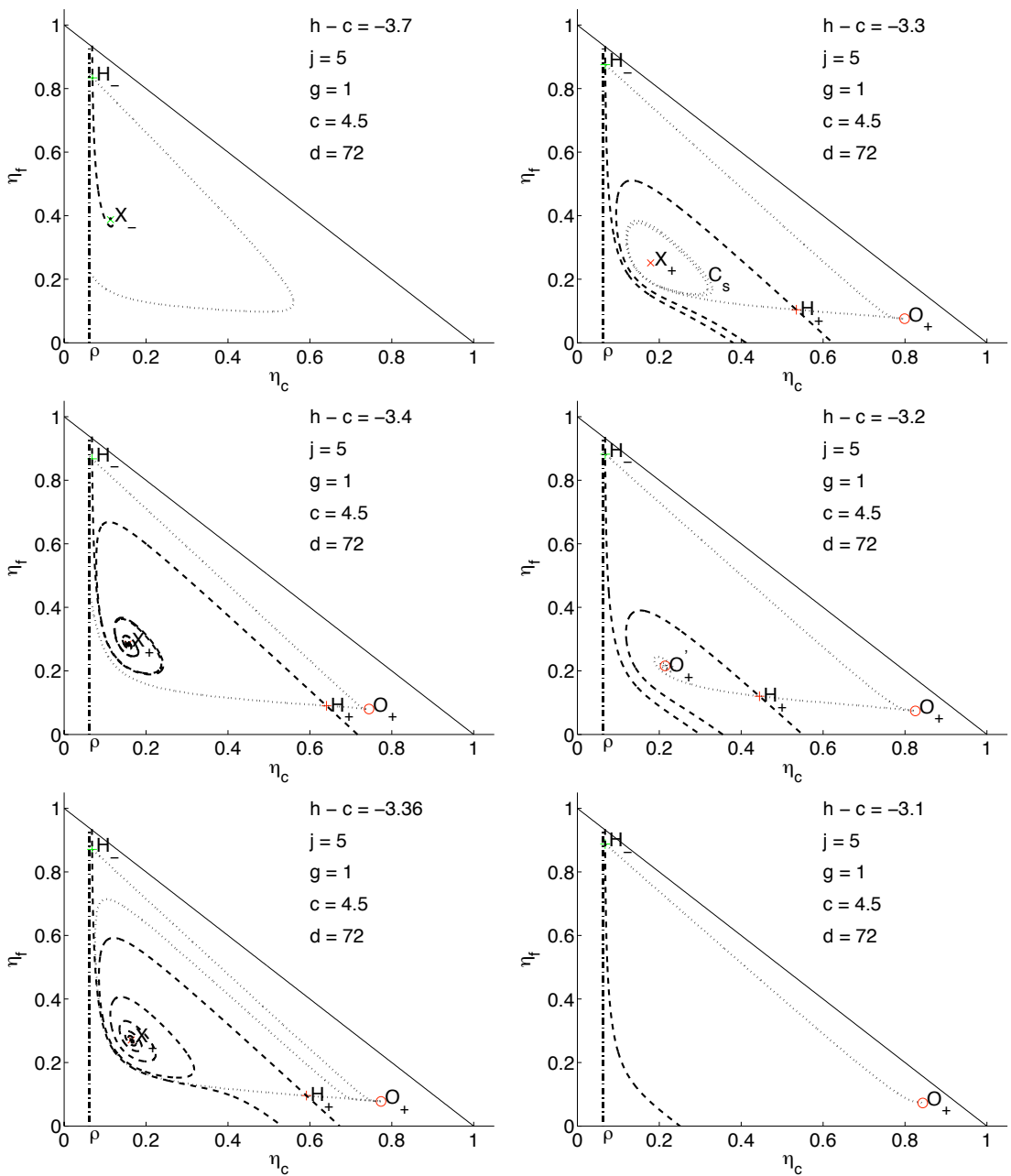

Figure 14: The stable and unstable manifolds for increasing values of $h-c$. For $h-c=-3.7$ only the trivial fixed points with $\eta_{c}=0$ exist. For $h-c=-3.4, H_{+}$ and $O_{+}$have been created from a saddle node bifurcation on $\eta_{1}^{+}(G)$. At $h-c=$ -3.36 a separatrix bifurcation between the stable manifold of $H_{-}$and the unstable manifold of $H_{+}$has introduced a first order transition in the basin of attraction. At $h-c=-3.3$ (reproduced from section 4) a separatrix bifurcation between the stable and unstable manifolds of $H_{+}$has introduced a stable cycle $C_{s}$ with its own basin of attraction (the interior of the line $C_{s}$ ). At $h-c=3.2$ (reproduced from section 4) the stable limit cycle $C_{s}$ has merged with the source $X_{+}$through a Hopf bifurcation to become a sink $O_{+}^{\prime}$. At $h-c=-3.1$ a final saddle node bifurcation has annihilated $O_{+}^{\prime}$ and $H_{+}$.

The algorithms used to compute the stable and unstable manifolds can 
be found in [20] and Chapter 10 of [21], respectively. The results classified according to the qualitative dynamical features are shown in figures (14). Hereafter we consider a system with parameters $j=5, g=1, c=4.5$, $d=72$, and provide the increasing values of $h-c$, denoted by $\lambda_{i}$, at which the successive bifurcations appear, together with the qualitative descriptions of their nature (figure 3 of section 4 summarizes the results).

- $\lambda_{1}=-4.0971$. For $h-c<\lambda_{1}$ there exists no intersection between $\eta_{1}(G)$ and $\eta_{2}(G)$ and all the points in $\mathbf{S}$ are eventually mapped to the trivial fixed point, which is always a solution of the equations. At $h-c=\lambda_{1}, \eta_{2}(G)$ begins to intersect $\eta_{1}^{-}(G)$ at two points, one of which is a saddle $\left(H_{-}\right)$and the other is a source $\left(X_{-}\right)$. The process of simultaneous creation or elimination of a pair of equilibrium points is called a saddle-node bifurcation. Note that this bifurcation does not alter the overall behavior of the system, since no new attractor is created. Thus, only the fixed points $\eta_{c}=0, \eta_{f}>0$ exist, and since $j>j_{B}$, the system may flow to either of the two fixed points for $\eta_{f}$ depending on the initial conditions.

- $\lambda_{2}=-3.4201$. Another saddle-node bifurcation happens on $\eta_{1}^{+}(G)$ at $h-c=\lambda_{2}$, with a saddle $\left(H_{+}\right)$and a sink $\left(O_{+}\right)$created simultaneously. Now the stable manifolds of $H_{+}$and $H_{-}$possess a common end emanating from $X_{+}$, and together they divide $\mathbf{S}$ into two regions. Points outside the region bounded by the stable manifolds should converge to the trivial fixed point $\eta_{c}=0, \eta_{f}>0$ as before, and those inside to $\mathrm{O}_{+}$.

- $\lambda_{3}=-3.3617$. For $\lambda_{2}<h-c<\lambda_{3}$, the unstable manifold of $H_{+}$ approaches the stable manifold of $H_{-}$until they coincide at $h-c=\lambda_{3}$ (the common manifold is called a separatrix between $H_{+}$and $H_{-}$). Beyond this value, the unstable manifold of $H_{+}$folds back and converges to $O_{+}$while the stable manifold of $H_{-}$hits the simplex boundary $\eta_{f}=0$. Now the basin boundary is determined solely by the stable manifold of $H_{-}$, causing the basin of attraction of $O_{+}$to experience a sudden expansion.

- $\lambda_{4}=-3.3504$. For $\lambda_{3}<h-c<\lambda_{4}$, the stable and unstable manifolds of $H_{+}$approach each other until they coincide at $h-c=\lambda_{4}$. Beyond this value, the former ends up at the boundary $\eta_{f}=0$ of $\mathbf{S}$ and the latter folds back into a stable limit cycle $C_{s}$ around $X_{+}$. At this transition $C_{s}$ is introduced into the system as a new attractor with its own basin of attraction delimited by the stable manifold of $H_{+}$.

- $\lambda_{5}=-3.2726$. For $\lambda_{4}<h-c<\lambda_{5}, C_{s}$ shrinks and eventually merges with $X_{+}$at $h-c=\lambda_{5}$ to produce a sink $O_{+}^{\prime}$. The process of transition 
between a sink and a source with the simultaneous appearance or disappearance of a limit cycle is called a Hopf bifurcation.

- $\lambda_{6}=-3.1146$. For $\lambda_{5}<h-c<\lambda_{6}, O_{+}^{\prime}$ and $H_{+}$approach each other until they are annihilated at $h-c=\lambda_{6}$. We are left with $H_{-}$and $\mathrm{O}_{+}$, the stable manifold of $H_{-}$serving as the basin boundary. This topology persists for arbitrarily larger values of $h-c$.

Summarizing the analysis above, we can identify three types of transition:

1. saddle-node bifurcation;

2. Hopf bifurcation;

3. separatrix bifurcation between two saddle points, or one saddle point with itself.

Type 1 can be directly determined from the number of intersections between $\eta_{1}(G)$ and $\eta_{2}(G)$ (cf. figure 2), or from the fact that $\mathbf{J}_{m}$ has an eigenvalue +1 . Due to the lack of symmetry in our system, transcritical and pitchfork bifurcations never occur ([17] $\S 3.4)$. In case that $\mathbf{J}_{m}$ has an eigenvalue -1 , one can have a period-doubling bifurcation $([17] \S 3.5)$. This never happens in our map, since one can easily verify that

$$
\mathbf{J}_{m}(1,1)>0, \quad \mathbf{J}_{m}(2,2)>0, \quad \Delta\left(\mathbf{J}_{m}\right)>0,
$$

which excludes the possibility that $\mathbf{J}_{m}$ has a negative eigenvalue.

Types 2 and 3 cannot be observed from the intersections between $\eta_{1}(G)$ and $\eta_{2}(G)$ and must be determined numerically. Type 2 remains a local bifurcation with $\mathbf{J}_{m}$ having a pair of conjugate complex eigenvalues with unit modulus. In contrast, Type 3 is a global bifurcation with the area of the basin of attraction experiencing a sudden jump or, in physicist's terms, a first-order transition. Numerically we have found no evidence for homoclinic intersection leading to chaos, but the question as to whether chaos exists in our two-dimensional map remains open.

Figures 15 are results of numerical simulations corresponding to the same parameters as in figure 14 .

\section{A.2 Phase structure of the flows}

We have computed the stable and unstable manifolds as for the map. The results are shown in Figures 16 (see figure 3 of section 4 for a summary). Hereafter we provide the bifurcation values of $h-c$, denoted by $\mu_{i}$, together with the qualitative descriptions of the nature of the bifurcation:

- $\mu_{1}=\lambda_{1}=-4.0971$. As in the parallel case, a saddle node bifurcation occurs at $h-c=\mu_{1}$ and produces a saddle $H_{-}$and a source $X_{-}$. 

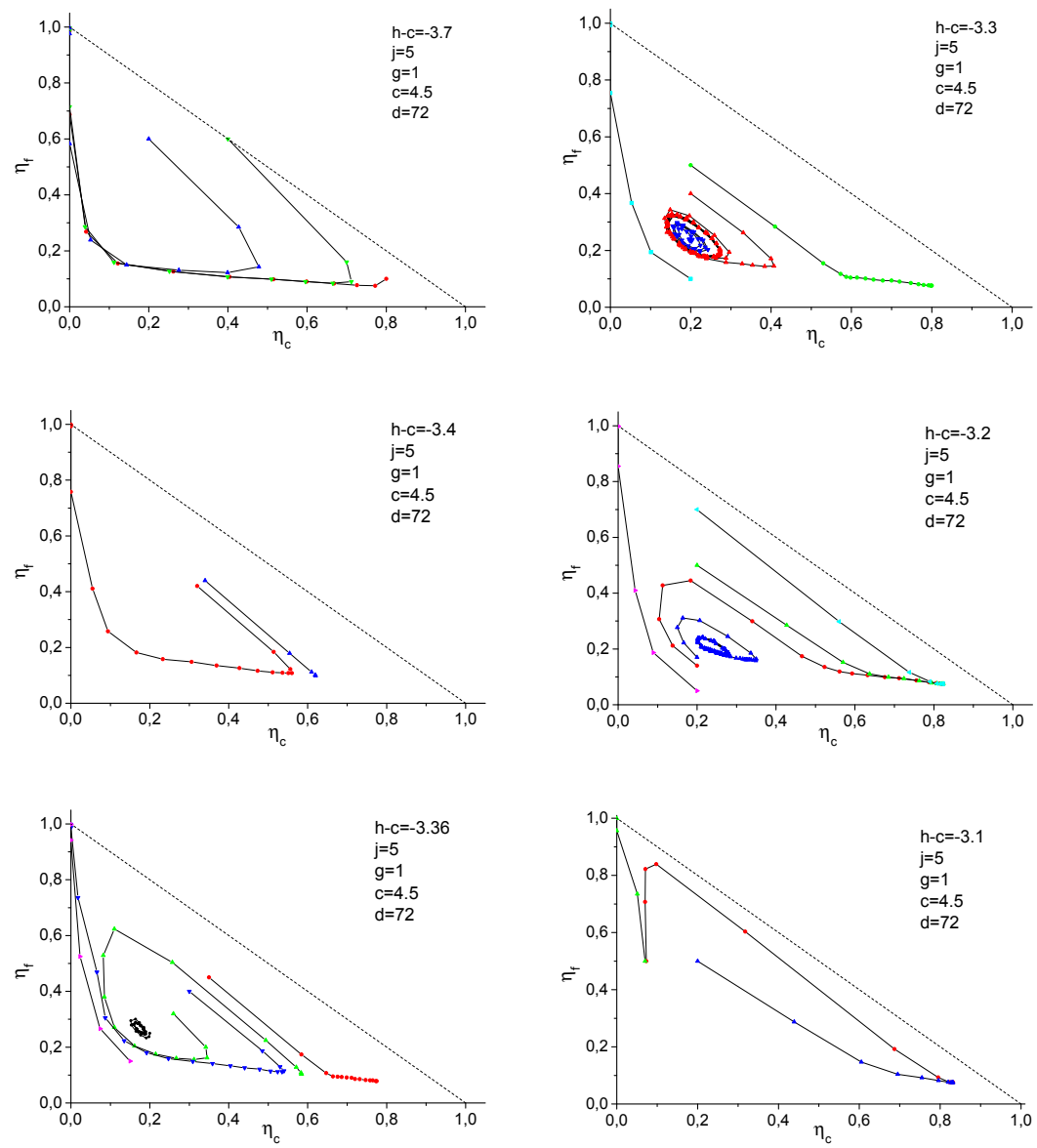

Figure 15: Examples of trajectories of a simulated system $(N=1000)$, starting from different initial conditions, corresponding to the maps of figure 14 . 

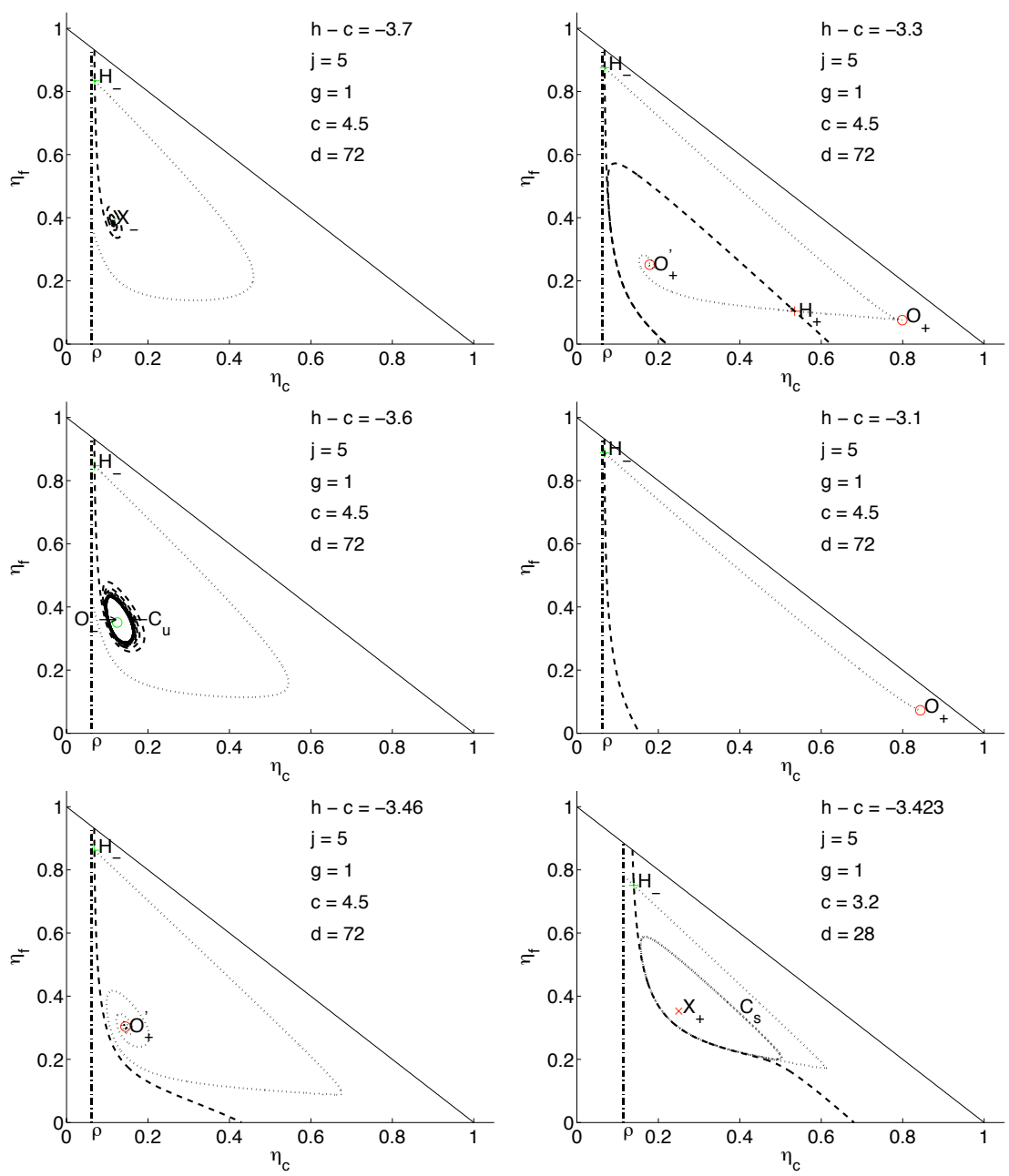

Figure 16: The stable and unstable manifolds for the flow for $h-c=3.7$ (Compare with figure 14 with the same value of $h-c$ ). At $h-c=-3.6$, a sink $O_{-}$together with an unstable limit cycle $C_{u}$ have emerged from the source $X_{-}$through a Hopf bifurcation. At $h-c=-3.46$ a separatrix bifurcation has eliminated $C_{u}$ and expanded the basin of attraction of the sink. At $h-c=-3.3, H_{+}$and $O_{+}$have been created from a saddle node bifurcation on $\eta_{1}^{+}(G)$ (Compare with figure 14 for the same value of $h-c$ ). For $h-c=-3.1$ a final saddle node bifurcation has annihilated $O_{+}^{\prime}$ and $H_{+}$(compare with Figure 14 for the same value of $h-c$ ). Notice that the figure on the bottom (right) shows a cycle that arises for other parameters $c$ and $d$. 
- $\mu_{2}=-3.6172$. A Hopf bifurcation occurs at $h-c=\mu_{2}$ and produces an unstable limit cycle $C_{u}$ together with a sink $O_{-}$from the source $X_{-}$. The basin of attraction of $O_{-}$is the area surrounded by $C_{u}$. Note that we've been able to choose $h-c$ such that the sink remains in the negative branch, which implies that variation of $\eta$ with respect to $h-c$ does not always qualify to determine the stability of the fixed point.

- $\mu_{3}=-3.4980$. A separatrix bifurcation of the stable and unstable manifolds of $H_{-}$eliminates $C_{u}$.

- $\mu_{4}=\lambda_{2}=-3.4201$. A saddle node bifurcation occurs at $h-c=\mu_{4}$ and produces a sink $O_{+}$and a saddle $H^{+}$.

- $\mu_{5}=\lambda_{6}=-3.1146$. A saddle node bifurcation occurs at $h-c=\mu_{5}$ and annihilates the saddle $H_{+}$and the source $O_{+}^{\prime}$. The topology persists for larger $h-c$.

Thus, we have the same types of bifurcation as in the parallel case, and Peixoto's Theorem ([17] Theorem 1.9.1) indicates that these are the only types of bifurcation expected in a two-dimensional flow system. However, let's note that the overall topology of the dynamics depends strongly on their order of occurrence. For example, if the separatrix bifurcation happens before the Hopf bifurcation, a stable limit cycle can be created just like in the parallel case (see the figure at the bottom (right) of figures 16). 